\title{
The measurement of structural and exchange income mobility
}

\author{
JAVIER RUIZ-CASTILLO \\ Departamento de Economí, Universidad Carlos III de Madrid, Madrid 126, Getafe 28903, \\ Madrid,Spain,E-mail:jrc@eco.uc3m.es
}

\begin{abstract}
Chakravarty, Dutta and Weymark (1985) present operational axioms for an ethical index of income mobility that are best suited for a two period world. This paper suggests a decomposition of this index into two terms: (i) an index of structural or snapshot mobility, which captures the welfare effect of differences in the inequality of the cross-section income distributions; and (ii) an index of exchange or rerankings mobility, which captures the welfare impact of rank reversals between the first and the second-period income distributions. Income inequality reductions and rank reversals are always welfare enhancing. The properties of all the income mobility concepts introduced in the paper do not require any new value judgements beyond the traditional ones.
\end{abstract}

Key words: distributional change, exchange and structural mobility, income mobility.

\section{Introduction}

Compared to an agrarian society, which has been the dominant social structure throughout most of history, our growth-oriented industrial society is presumed to be socially mobile and egalitarian. The recent availability of longitudinal data makes the measurement of such a central concept as mobility increasingly possible. In the words of the authors of a recent survey, the problem is that, compared with the neighboring area of inequality measurement, "the income mobility literature is still distressingly far from being unifie on how to measure mobility and make mobility comparisons", Fields and Ok [8, p. 586], - or FO for short, from here on.

Among the many issues that remain open, the main question addressed in this paper is the distinction between structural and exchange mobility. ${ }^{1}$ To study under what conditions structural, exchange and total mobility are socially desirable, this paper uses ethical or normative mobility indexes. More precisely, it follows the ethical approach originally suggested in Chakravarty, Dutta and Weymark [3], or CDW for short. They compare the actual time path of incomes received over a number of periods with a hypothetical income structure that captures the notion of complete immobility. For operational reasons, CDW make two key assumptions. First, complete immobility is taken to mean constancy over time of the relative or absolute positions occupied by the individuals in the actual first-perio income 
distribution. Second, the SEF is define on the aggregate incomes received by each individual over all the time periods under consideration. This model is best seen to apply to a two period world. ${ }^{2}$

In this framework, it is essential to distinguish between two types of rank reversals ignored in CDW: rank reversals between the first and second-period income distributions, which will be called 1/2-rerankings; and rank reversals between the first-perio and the aggregate income distributions, which will be called $1 / 1+$ 2-rerankings. The distinction can be illustrated by means of a pair of simple examples for an economy with two individuals. In both examples the firs period income distribution is $(2,4)$. In Example 1, the second period income distribution is $(4,3)$. Therefore, there is a $1 / 2$-reranking; but since the aggregate income distribution is $(6,7)$, there is no $1 / 1+2$-reranking. In Example 2, the second period income distribution is $(7,0)$, representing the same total income growth as before. The aggregate income distribution is now $(9,4)$, so there is both a $1 / 2-$ and a $1 / 1+2$-reranking.

Using this distinction, a novel decomposition of CDW's mobility index is offered, where overall income mobility is expressed as the sum of two terms: the firs one, called structural or snapshot mobility, captures the welfare effect of the change in income inequality between the aggregate and the completely immobile distribution, once all 1/2-rerankings have been eliminated. Income inequality reductions between the first and the second-period income distributions cause structural mobility to be positive. The second term, called exchange or rerankings mobility, measures the welfare impact of 1/2-rerankings, with or without $1 / 1+2$-rerankings. ${ }^{3}$ No value judgments are imposed either on $1 / 2$ - or $1 / 1+2$ rerankings. However, in the presence of the firs type of rank reversals, it is shown that exchange mobility is always socially desirable.

The rest of the paper is organized in three sections. Section 2 presents the assumptions of the CDW model in a two period world. Section 3 contains the decomposition of the income mobility into structural and exchange mobility, while Section 4 concludes.

\section{The model}

The approach followed in this paper is meant for a setting in which the variable of interest is income. Abstracting from the transition mechanism, the focus is on the changes that can be observed in a straightforward way in longitudinal data sets: changes in cross-section or snapshot income inequality, and changes in relative incomes or in absolute income differences. In this context, an index of mobility is define as a real-valued function on the set of time paths of income distributions. Indices of relative or absolute mobility are sensitive to changes in relative incomes or in income differences, respectively. ${ }^{4}$

Let there be $n \geq 2$ individuals, indexed by $i=1, \ldots, n$, and let $D$ be the non-negative orthant in $n$-dimensional Euclidean space with the origin removed. 
In a two period world, let $\mathbf{x}=\left(x^{1}, \ldots, x^{n}\right) \in D$ represent the income distribution of an $n$-person society where individual $i$ 's income level is $x^{i}$. Now assume that individual $i$ 's income has changed to $y^{i}$ in a given time interval. Following FO's terminology, it is said that $\mathbf{x}$ has been transformed to $\mathbf{y}=\left(y^{1}, \ldots, y^{n}\right) \in D$, and this so-called distributional transformation is denoted by $\mathbf{x} \rightarrow \mathbf{y}$. In what follows, in every distributional transformation $\mathbf{x} \rightarrow \mathbf{y}$, income distribution $\mathbf{x}$ will be ordered according to the "less than or equal" relation. Each individual $i$ is characterized by an income stream $\left(x^{i}, y^{i}\right)$. Over the two periods, individual $i$ receives aggregate income $x^{i}+y^{i}$. The distribution $\mathbf{x}+\mathbf{y}=\left(x^{1}+y^{1}, \ldots, x^{n}+y^{n}\right)$ is referred to as the aggregate income distribution.

As pointed out by FO, while it admittedly confine the analysis to only 2period paths of income distributions, this framework permits to study both intra and intergenerational mobility measurement, depending on the length of the time period between the observation periods. In the intergenerational case, assume that every parent has only one child. Then, $x^{i}$ and $y^{i}$ can be interpreted as the parent and the child incomes, respectively, and $x^{i}+y^{i}$ as the dynastic or family income. In the intragenerational case, $x^{i}$ and $y^{i}$ can be taken to be individual $i$ 's income while "young" and "old", respectively, and $x^{i}+y^{i}$ his/her lifetime income. Therefore, as in the seminal papers by Markandya [11, 12] and King [10], the next two sections of the paper will be restricted to this particular world.

The ethical approach to measuring income mobility in CDW uses an intertemporal social evaluation function (SEF for short), $F: D^{2} \rightarrow R^{1}$, where $F(\mathbf{x}, \mathbf{y})$ is the social welfare level associated with the distributional transformation $\mathbf{x} \rightarrow \mathbf{y}$. The income mobility concept actually explored is the one embodied in a welfare comparison of the actual distributional transformation $\mathbf{x} \rightarrow \mathbf{y}$, and a hypothetical benchmark $\mathbf{x} \rightarrow \mathbf{v}$ : the distributional transformation which would have resulted in the absence of mobility given the firs period distribution $\mathbf{x}$. That is to say, mobility indices are obtained by comparing the actual level of social welfare $F(\mathbf{x}, \mathbf{y})$ with the level of social welfare $F(\mathbf{x}, \mathbf{v})$ which would have been obtained with the benchmark distributional transformation $\mathbf{x} \rightarrow \mathbf{v}$.

Let $\mu(\mathbf{x})$ be the mean of any income distribution $\mathbf{x} \in D$. In the relative case, $\mathbf{v}$ is define by $\mathbf{v}=(\mu(\mathbf{y}) / \mu(\mathbf{x})) \mathbf{x}$. Thus, the aggregate distribution in the benchmark transformation, $\mathbf{x}+\mathbf{v}$, has the same mean as $\mathbf{x}+\mathbf{y}$ but individual income shares are maintained equal to the income shares in the firs period distribution $\mathbf{x}$, i.e. $\mu(\mathbf{v})=\mu(\mathbf{y}), \mu(\mathbf{x}+\mathbf{v})=\mu(\mathbf{x}+\mathbf{y})$, and $I(\mathbf{v})=I(\mathbf{x})=I(\mathbf{x}+\mathbf{v})$ for any index of relative inequality $I$.

To make the comparison between $F(\mathbf{x}, \mathbf{y})$ and $F(\mathbf{x}, \mathbf{v})$ operational, CDW assume that the only features of the distributional transformations $\mathbf{x} \rightarrow \mathbf{y}$ and $\mathbf{x} \rightarrow \mathbf{v}$ relevant for the welfare comparisons are their aggregate distributions $\mathbf{x}+\mathbf{y}$ and $\mathbf{x}+\mathbf{v}$. Formally:

A.1. There exists a SEF $W: D \rightarrow R^{1}$ such that, for all distributional transformations $\mathbf{x} \rightarrow \mathbf{y}, W(\mathbf{x}+\mathbf{y})=F(\mathbf{x}, \mathbf{y})$. Moreover, $W(D) \subseteq R_{++}$. 
An income mobility index assigns a mobility value to each distributional transformation $\mathbf{x} \rightarrow \mathbf{y}$, i.e. it is a function $M: D^{2} \rightarrow R^{1}$. CDW suggest the following index of income mobility in the relative case:

$$
M(\mathbf{x}, \mathbf{y})=\{W(\mathbf{x}+\mathbf{y})-W(\mathbf{x}+\mathbf{v})\} / W(\mathbf{x}+\mathbf{v}) .
$$

An immobile income structure is assigned a mobility value of zero.

The next assumption, which is also taken from CDW, refers to the welfare evaluation of one-period income distributions.

A.2. There exists a SEF define on one-period incomes, $W^{*}: D \rightarrow R^{1}$, and this function is the same as the two-period SEF $W$.

The identificatio of one-period evaluations with two-period ones is questionable, but it greatly simplifie our work. ${ }^{5}$ The remaining properties of the income mobility index depend on additional assumptions on the SEF. For analytical purposes, it is only needed that $W$ can be expressed in terms of only two statistics of the income distribution, the mean and an index of income inequality. However, for operational purposes it is convenient to specify the trade-off between efficien y and distributional considerations. Consequently, in the relative case the following assumption is made:

A.3. For any income distribution $\mathbf{x} \in D$, the SEF $W$ can be expressed as: $W(\mathbf{x})=$ $\mu(\mathbf{x})(1-I(\mathbf{x}))$. That is, social welfare is seen to be the product of the mean times an adjustment factor that varies inversely with an appropriate index (i.e. a continuous, S-convex and scale invariant index) of relative inequality $I .{ }^{6}$

In this case, the CDW income mobility index define in (1) becomes

$$
M(\mathbf{x}, \mathbf{y})=\{I(\mathbf{x})-I(\mathbf{x}+\mathbf{y})\} /\{1-I(\mathbf{x})\} .
$$

Contrary to descriptive income mobility indices, this ethical index allows us to determine whether the observed income movement is socially desirable. Consider the following two examples:

$\mathrm{E} 1: \mathbf{x}=(2,4) \rightarrow \mathbf{y}=(4,3) ; \mathbf{x}+\mathbf{y}=(6,7)$,

$\mathrm{E} 2: \mathbf{x}=(2,4) \rightarrow \mathbf{y} \#=(2,5) ; \mathbf{x}+\mathbf{y} \#=(4,9)$.

The initial situation is the same in both examples, $\mathbf{x}=(2,4)$. Since $\mu(\mathbf{y})=$ $\mu(\mathbf{y} \#)=7 / 2$, the rate of income growth is also the same in E1 and E2. However, it is clear that $M(\mathbf{x}, \mathbf{y})=\{I(2,4)-I(6,7)\} /\{1-I(2,4)\}>0$, while $M(\mathbf{x}, \mathbf{y} \#)=\{I(2,4)-I(4,9)\} /\{1-I(2,4)\}<0$. The reduction in income inequality in $\mathbf{x}+\mathbf{y}$ relative to $\mathbf{x}$ causes $M(\mathbf{x}, \mathbf{y})$ to be positive, reflectin an increase in social welfare. The opposite situation causes $M(\mathbf{x}, \mathbf{y} \#)$ to be negative, reflectin a social welfare loss. Thus, the social value of income mobility in this approach is rooted in an aversion to aggregate income inequality.

Notice that a distributional transformation $\mathbf{x} \rightarrow \mathbf{y}$ that involves only a change in scale causes no mobility in the relative case, i.e. whenever $\mathbf{y}=\lambda \mathbf{x}$ for some $\lambda>0$, 
$M(\mathbf{x}, \lambda \mathbf{x})=0$. In other words, in this approach income growth per se has no mobility consequences. For $M(\mathbf{x}, \mathbf{y}) \neq 0$, it is necessary that either $I(\mathbf{x}) \neq I(\mathbf{y})$ or that there is some 1/2-reranking, so that $I(\mathbf{x})$ can be different from $I(\mathbf{x}+\mathbf{y})$. When this is the case, differences in mean incomes do affect social welfare and hence income mobility, but only through their impact on $I(\mathbf{x}+\mathbf{y})$. The conclusion is that, in this framework, income mobility and income growth remain largely independent concepts.

Moreover, in FO's terminology the CDW index define in Equation (2) is weakly relative, i.e. for any income transformation $\mathbf{x} \rightarrow \mathbf{y}, M(\lambda \mathbf{x}, \lambda \mathbf{y})=\{I(\lambda \mathbf{x})-$ $I(\lambda(\mathbf{x}+\mathbf{y}))\} /\{1-I(\lambda \mathbf{x})\}=\{I(\mathbf{x})-I(\mathbf{x}+\mathbf{y})\} /\{1-I(\mathbf{x})\}=M(\mathbf{x}, \mathbf{y})$ for all $\lambda>0 .{ }^{7}$ Finally, notice that this mobility index is sensitive to the choice of the base period. However, in a two period world this is not important because the choice of base period is naturally given both in the intergenerational and the intragenerational interpretations: the income distribution of the parent generation and the "young", respectively. ${ }^{8}$

\section{Structural and exchange mobility}

\subsection{DEFINITIONS}

Apparently, the income mobility index define in Equation (2) reflect welfare changes due solely to changes in income inequality from the initial to the fina situation. One of the points of this paper is to clarify why this is not the case at all. Upon closer inspection, income changes in Example E1 presented in the previous section give rise to two effects. First, a change in cross-section or snapshot income inequality from $I(2,4)$ to $I(4,3)$. Second, a rank reversal of individual incomes between the first and the second-period income distributions, that is to say, a 1/2-reranking: in distribution $\mathbf{x}$ individual 1 is poorer than 2, while in distribution $\mathbf{y}$ individual 1 is richer.

At this point, it is useful to consider a third example:

E3: $\mathbf{x}=(2,4) \rightarrow \mathbf{y}^{*}=(5,2) ; \mathbf{x}+\mathbf{y}^{*}=(7,6)$.

Both the initial situation and the rate of income growth coincide with those of Examples E1 and E2. Given the symmetry of $I$, we have $I(6,7)=I(7,6)$. Hence, $M\left(\mathbf{x}, \mathbf{y}^{*}\right)=M(\mathbf{x}, \mathbf{y})$. The novelty in relation to E1, is that in E3 there is both a $1 / 2$ - and a $1 / 1+2$-reranking. Of course, any $1 / 1+2$-reranking implies some $1 / 2$-reranking (as in E3), but not the contrary (as in E1).

Examples E1 and E3 suggest that CDW's mobility index can be decomposed into two terms. One capturing the welfare change due to the change in inequality between the cross-section distributions $\mathbf{x}$ and $\mathbf{y}$ once all 1/2-rerankings have been removed, and a second one capturing the 1/2-rerankings effect with or without $1 / 1+2$-rerankings between $\mathbf{x}$ and $\mathbf{x}+\mathbf{y}$. Therefore, from a formal point of view what is desired is a decomposition of the mobility index $M(\mathbf{x}, \mathbf{y})$ into a structural 
or snapshot mobility index, $S M(\mathbf{x}, \mathbf{y})$, and an exchange or rerankings mobility component, $\operatorname{EM}(\mathbf{x}, \mathbf{y})$.

Given any distributional transformation $\mathbf{x} \rightarrow \mathbf{y}$, def ne $\tilde{\mathbf{y}}$ as having the same components as $\mathbf{y}$, but rearranged (if necessary) in the same increasing order as $\mathbf{x}$. The following decomposition of the mobility index is now introduced:

$$
M(\mathbf{x}, \mathbf{y})=S M(\mathbf{x}, \mathbf{y})+E M(\mathbf{x}, \mathbf{y}),
$$

where

$$
\begin{aligned}
S M(\mathbf{x}, \mathbf{y}) & =\{W(\mathbf{x}+\tilde{\mathbf{y}})-W(\mathbf{x}+\mathbf{v})\} / W(\mathbf{x}+\mathbf{v}) \\
& =\{I(\mathbf{x})-I(\mathbf{x}+\tilde{\mathbf{y}})\} /\{1-I(\mathbf{x})\}, \\
E M(\mathbf{x}, \mathbf{y}) & =\{W(\mathbf{x}+\mathbf{y})-W(\mathbf{x}+\tilde{\mathbf{y}})\} / W(\mathbf{x}+\mathbf{v}) \\
& =\{I(\mathbf{x}+\tilde{\mathbf{y}})-I(\mathbf{x}+\mathbf{y})\} /\{1-I(\mathbf{x})\} .
\end{aligned}
$$

The term $\operatorname{SM}(\mathbf{x}, \mathbf{y})$ can be viewed as the income mobility associated with the distributional transformation $\mathbf{x} \rightarrow \tilde{\mathbf{y}}$ in which all the 1/2-rerankings between $\mathbf{x}$ and $\mathbf{y}$ have been eliminated, i.e. $S M(\mathbf{x}, \mathbf{y})=M(\mathbf{x}, \tilde{\mathbf{y}})$. Then, exchange mobility is define as a residual, i.e. $E M(\mathbf{x}, \mathbf{y})=M(\mathbf{x}, \mathbf{y})-M(\mathbf{x}, \tilde{\mathbf{y}}){ }^{9}$

\subsection{PROPERTIES}

Remark 1. Recall that $I(\tilde{\mathbf{y}})=I(\mathbf{y})$. Since $\mathbf{x}, \tilde{\mathbf{y}}$, and hence $\mathbf{x}+\tilde{\mathbf{y}}$ are equally ordered, it is clear that $I(\mathbf{x}+\tilde{\mathbf{y}})$ cannot be larger than $\operatorname{Max}\{I(\mathbf{x}), I(\mathbf{y})\}$. Therefore, for any $\mathbf{x}, \mathbf{y} \in D$ which are not scalar multiples of each other,

$$
I(\mathbf{x}) \geq I(\mathbf{y}) \Rightarrow S M(\mathbf{x}, \mathbf{y}) \geq 0 .
$$

Thus, whenever $I(\mathbf{x})>I(\mathbf{y})$ the structural mobility index captures the welfare increase due to a decrease in cross-section or snapshot inequality. However, as shown by the following example, the converse to Equation (4) does not hold. Let $\mathbf{x}=(1,2,3), \mathbf{y}=(1.21,2.00585,3.38415), \mathbf{x}+\mathbf{y}=(2.21,4.00585,6.38415)$, and use the coefficien of variation, $C$, as an index of income inequality. Then $C(\mathbf{x})=0.5>C(\mathbf{x}+\mathbf{y})=0.498533$, so that $S M(\mathbf{x}, \mathbf{y})>0$ but $C(\mathbf{x})<C(\mathbf{y})=$ $0.500001 .{ }^{10}$

On the other hand, consider the case in which there is no 1/2-reranking, so that $\tilde{\mathbf{y}}=\mathbf{y}$. As pointed out in FO, in King's [10] model there is no mobility. In our case, all mobility is structural mobility and, as long as $I(\mathbf{x}) \neq I(\mathbf{y})$, mobility is in general different from zero.

In the presence of some 1/2-rerankings, it can be shown that exchange mobility is always socially desirable.

THEOREM 1. Let $\mathbf{x} \rightarrow \mathbf{y}$ be a distributional transformation such that $\tilde{\mathbf{y}} \neq \mathbf{y}$, i.e. such that there is some 1/2-rerankings. Then, $\operatorname{EM}(\mathbf{x}, \mathbf{y})>0$. 
(The proof is a direct consequence of Proposition A.1 in Marshall and Olkin [13, p. 139], taken from Day [5].)

If $I(\mathbf{x})=I(\mathbf{y})$, then there is only exchange mobility. If, however $I(\mathbf{x})>I(\mathbf{y})$, as in Example E1, then structural mobility is positive. In either case, total mobility $M(\mathbf{x}, \mathbf{y})$ will be positive. When $I(\mathbf{x})<I(\mathbf{y})$ and, contrary to the example in Remark 1, $S M(\mathbf{x}, \mathbf{y})<0$, then the sign of $M(\mathbf{x}, \mathbf{y})$ depends on the relative strength of $\operatorname{SM}(\mathbf{x}, \mathbf{y})$ and $\operatorname{EM}(\mathbf{x}, \mathbf{y})$. Consider the following example:

$\mathrm{E} 4: \mathbf{x}=(2,4) \rightarrow \mathbf{y}^{\circ}=(7,0) ; \mathbf{x}+\mathbf{y}^{\circ}=(9,4)$.

There is a $1 / 1+2$-reranking and, therefore, a 1/2-reranking which causes $E M\left(\mathbf{x}, \mathbf{y}^{\circ}\right)>0$. On the other hand, since $I(\mathbf{x})=I(2,4)<I\left(\mathbf{x}+\mathbf{y}^{\circ}\right)=I(2,11)$ we have that $S M\left(\mathbf{x}, \mathbf{y}^{\circ}\right)<0$. It turns out that $I(\mathbf{x})<I\left(\mathbf{x}+\mathbf{y}^{\circ}\right)=(9,4)$, so that $M\left(\mathbf{x}, \mathbf{y}^{\circ}\right)<0$, i.e. $\operatorname{SM}\left(\mathbf{x}, \mathbf{y}^{\circ}\right)$ is greater in absolute value than $\operatorname{EM}\left(\mathbf{x}, \mathbf{y}^{\circ}\right)$.

\section{Conclusions}

Until recently, the evaluation of an economy's performance over time was only possible with individual income data drawn from two (or more) independent population samples in different time periods. It is quite obvious that the usual comparison in inequality or social welfare in terms of two snapshot income distributions permits only a partial evaluation of the dynamic economic process. A convincing criticism is conveniently summarized in the following quotation from Karcher et al. [9]: “... since the individuals' positions on the cardinal income scale rarely remain unchanged over time, an increase in a snapshot measure of inequality is clearly consistent with there having been a significan amount of equalizing mobility over time. Only if all individuals' earnings remain constant from period to period will a measure of inequality or welfare give the same result irrespective of the length of the accounting period".

CDW's modeling of the dynamic evaluation problem can be viewed as an attempt to confront this criticism. Conceptually, they suggest that an income mobility index can be obtained by comparing the social welfare of the actual distributional transformation with the social welfare of a hypothetical distributional transformation that exhibits no mobility. Operationally, they assume that the social welfare of any distributional transformation can be identifie with the social welfare of the aggregate income distribution. In particular, under the type of SEF adopted in this paper, what matters at the social level is the inequality of the aggregate income distribution - a fundamental idea implicit in the previous quotation.

There are several observable factors in longitudinal data sets that affect the inequality of the aggregate income distribution. What has been done in this paper is to develop the idea that, within CDW's framework in a two period world, it is useful to distinguish between changes in cross-section or snapshot income inequality, and changes in ranks. Accordingly, it has been shown how to decompose CDW's 
income mobility index into two indexes of structural and exchange mobility which capture, respectively, the welfare effect of these two types of changes.

From a conceptual point of view, it should be emphasized that all the properties of the different income mobility concepts introduced in this paper have been obtained in a framework which, contrary to the seminal contributions of King [10] and Markandya [11, 12], does not involve any new value judgments beyond the traditional ones. In particular, the fact that rank reversals resulting from income mobility have a positive effect on social welfare is a consequence rather than an assumption of the model.

In situations where there are individual rerankings, the approach presented in this paper is immediately applicable. ${ }^{11}$ On the other hand, whether the decomposition developed is useful in other income mobility models is an open question left for future research.

\section{Acknowledgements}

Financial help from Fundación Argentaria's Programa de Igualdad and Project PB96-0118 from the Spanish DGICYT is gratefully acknowledged. The paper has been presented in the European network Living Standards, Inequality, and Taxation, finance by the ECC TMR Contract \#ERBFMRXCT980248. I wish to acknowledge many conversations with Carmen Vargas and Francisco Marhuenda. Comments from the editor and two referees' reports led to substantial improvements of the paper. However, all remaining shortcomings are my sole responsibility.

\section{Notes}

1 As FO conclude, "All in all, the present literature on income mobility falls short of providing an exact, robust decomposition of total mobility into its basic sources" (p. 565). For another valuable discussion of the difficultie involved in modeling structural and exchange mobility, see Shorrocks [19].

${ }^{2}$ See Markandya [11, 12] and King [10] for alternative concepts of income mobility in a two period horizon.

${ }^{3}$ It must be admitted that there is no consensus about what 'structural' or 'exchange' mobility mean. In any case, the reader can always refer to the concepts introduced in this paper as 'snapshot' or 'rerankings' mobility.

${ }^{4}$ For descriptive measures in this framework, see inter alia the relative indices suggested by Shorrocks [18] and Cowell [4], and the absolute indices proposed by Berrebi and Silber [1] and Fields and Ok $[6,7]$.

${ }^{5}$ Shorrocks [17] justifie A.2 as a direct application in the intertemporal context of the population replication axiom, usually assumed in income distribution theory in order to compare the income inequality of populations of different size.

${ }^{6}$ For example, CDW study the case in which $W(\mathbf{x})=\mu(\mathbf{x})\left\{1-I^{\mathrm{AKS}}(\mathbf{x})\right\}$, where $I^{\mathrm{AKS}}$ is the inequality index obtained according to the Atkinson-Kolm-Sen procedure which uses the notion of an equally distributed income. As it is well known, whenever $W$ is homothetic $I^{\mathrm{AKS}}$ is a scale 
invariant inequality index. Alternatively, because of its nice additive separability properties, RuizCastillo [15] uses $W(\mathbf{x})=\mu(\mathbf{x})\left\{1-I_{C}(\mathbf{x})\right\}$, where $I_{C}$ for $c=1$, 2, stand for the following members of the general entropy family of income inequality indices: the firs inequality index originally suggested by Theil [20], and an ordinal transformation of the coefficien of variation, respectively.

${ }^{7}$ On the other hand, given a distributional transformation $\mathbf{x} \rightarrow \mathbf{y}, M(\lambda \mathbf{x}, \alpha \mathbf{y}) \neq M(\mathbf{x}, \mathbf{y})$ for different $\lambda$ and $\alpha>0$, i.e. in FO's terminology the income mobility index explored in this paper is not strongly relative or intertemporally scale invariant.

${ }^{8}$ It is easy to show that the consequences of the base period reversal depend on the relation between $I(x)$ and $I(y)$. Specificall,$M(\mathbf{x}, \mathbf{y})>=<M(\mathbf{y}, \mathbf{x}) \Leftrightarrow I(\mathbf{x})>=<I(\mathbf{y})$.

${ }^{9}$ In this sense, this approach follows Definitio II in Markandya [12].

${ }^{10}$ I owe this example to John Weymark.

${ }^{11}$ In this respect, see CDW's reinterpretation of Blackorby and Donaldson [12] tax-benefi model. Consider also the possibility, as in Ruiz-Castillo and Sastre [16], of interpreting $\mathbf{x}$ and $\mathbf{y}$ as the husbands' and wives' income distributions, respectively. Finally, for an analysis of the income mobility induced by the income tax, as well as the measurement of horizontal inequality as the extent of rank reversals between the pre-tax and the after-tax income distributions, see Section 3 in Ruiz-Castillo [14].

\section{References}

1. Berrebi, Z. and Silber, J.: On an absolute measure of distributional change, European Economic Review 22 (1983), 139-146.

2. Blackorby, C. and Donaldson, D.: Ethical social index numbers and the measurement of effective tax/benefi progressivity, Canadian Journal of Economics XVII (1984), 683-694.

3. Chakravarty, S., Dutta, B. and Weymark, J.: Ethical indices of income mobility, Social Choice and Welfare 2 (1985), 1-21.

4. Cowell, F.A.: Measures of distributional change: An axiomatic approach, Review of Economic Studies 52 (1985), 135-151.

5. Day: Rearrangement inequalities, Canadian Journal of Mathematics 24 (1972), 930-943.

6. Fields, G. and Ok, E.: The meaning and measurement of income mobility, Journal of Economic Theory 71 (1996), 349-377.

7. Fields, G. anf Ok, E.: Measuring movement of incomes, Economica 66 (1999), 455-471.

8. Fields, G. and Ok, E.: The measurement of income mobility: An introduction to the literature, In: J. Silber (ed.), Handbook on Income Inequality Measurement Kluwer Academic Publishers, Dordrecht, 2000.

9. Karcher, T., Moyes, P. and Trannoy, A.: The stochastic dominance ordering of income distributions over time: The discounted sum of the expected utilities of incomes, In: W.A. Barnett, H. Moulin, M. Salles and N.J. Schofiel (eds.), Social Choice, Welfare and Ethics Cambridge University Press, Cambridge, 1995, Chapter 17, pp. 375-408.

10. King, M.: An index of inequality: With applications to horizontal equity and social mobility, Econometrica 51 (1983), 99-115.

11. Markandya, A.: Intergenerational exchange mobility and economic welfare, European Economic Review 17 (1982), 307-324.

12. Markandya, A.: The welfare measurement of changes in economic mobility, Economica $\mathbf{5 1}$ (1984), 457-471.

13. Marshall, A.W. and Olkin, I.: Inequality: Theory and Majorization and Its Applications, Academic Press, New York, 1979.

14. Ruiz-Castillo, J.: Income mobility, permutations and rerankings, Working Paper 97-74, Universidad Carlos III de Madrid, 1997. 
15. Ruiz-Castillo, J.: The measurement of inequality of opportunities, forthcoming, In: J. Bishop and Y. Amiel (eds.), Research in Economic Inequality, Vol. 9, 2003, pp. 1-34.

16. Ruiz-Castillo, J. and Sastre, M.: Distributive implications of member level income aggregation within the household: An approximation through income mobility indexes, Economics of Transition 9 (2000), 487-513.

17. Shorrocks, A.F.: The measurement of mobility, Econometrica 46 (1978), 1013-1024.

18. Shorrocks, A.F.: Income inequality and income mobility, Journal of Economic Theory $\mathbf{1 0}$ (1978), 376-393.

19. Shorrocks, A.: On the Hart measure of income mobility, In: M. Carson, J. Creedy (eds.), Industrial Concentration and Economic Inequality, Edward Elgar, 1993.

20. Theil, H.: Economics and Information Theory, North-Holland, Amsterdam, 1967. 